\title{
Recycling of vinasse in ethanol fermentation and application in Egyptian distillery factories
}

\author{
Fadel, M. ${ }^{1 *}$, Abdel-Naser A. Zohri ${ }^{2}$, M. Makawy ${ }^{3}$, M. S. Hsona ${ }^{3}$ and A. M. Abdel-Aziz ${ }^{3}$ \\ ${ }^{1}$ Microbial Chemistry Department, National Research Center, Cairo, Egypt. \\ ${ }^{2}$ Botany and Microbiology Department, Faculty of Science, Assiut University, Egypt. \\ ${ }^{3}$ Distillation Factories, Sugar and Integrated Company, El- Hawamdia Giza, Egypt.
}

Received 1 August, 2014; Accepted 13 October, 2014

\begin{abstract}
Vinasse production is one of the most significant and challenging issues in the industrial production of ethanol due to pollution problems. In this study, vinasse obtained from ethanol by fermentation of sugarcane molasses was used instead of water to prepare the fermentation medium. Saccharomyces cerevisiae F-727 was selected from 10 tested yeast strains. Nutritional requirements of nitrogen, phosphorous and magnesium, as well as the effect of inoculum size were studied. There was a decrease in required nutrients when vinasse was used instead of fresh water; and $\mathbf{5 0 \%}$ yield was achieved in fresh water together with an improved efficiency of the fermentation.
\end{abstract}

Key words: Vinasse, ethanol fermentation, Saccharomyces cerevisiae.

\section{INTRODUCTION}

The disposal of vinasse, the major effluent from the ethanol industry, represents a major environmental problem for the ethanol industry. This black liquid produced 10 to 15 times greater than the ethanol itself is a mixture of water and organic and inorganic compounds. These compounds remain after different steps involving sugar cane production and processing. These hazardous substances cause the vinasse to have a very high biological oxygen demand (BOD), ranging from 3040,000 (Voegele, 2009). The inadequate and indiscriminate disposal of sugarcane vinasse in soils and water bodies has received much attention since decades ago, due to environmental problems associated to this practice (Aparecida et al., 2013).

Research has demonstrated that vinasse disposal in river basins alone is not a convenient disposal solution. Due to its high B.O.D., this material can cause damage to aquatic life, especially when dumped in large volumes. Some of the existing methods for the disposal of vinasse are direct land application and methane production (Baez-Smith, 2006). If vinasse is discharged on land, the alkalinity of the soil is reduced, leading to destruction of crops, deficiency of manganese in the soil and inhibition of seed germination. Also, the concentration-incineration of vinasse is the only system that can provide a satisfactory solution to the pollution problem; its only draw-back is high cost (Aparecida et al., 2013). Due to the large quantities of vinasse produced, alternative treatments and uses have been developed, such as recycling of vinasse in fermentation, fertirrigation,

*Corresponding author. E-mail: mfadel54@yahoo.com.

Author(s) agree that this article remain permanently open access under the terms of the Creative Commons Attribution License 4.0 $\underline{\text { International License }}$ 
Table 1. Screening S. cerevisiae strains cultivated on sugar cane molasses medium diluted with $50 \%$ vinasse instead of water.

\begin{tabular}{lccc}
\hline Yeast strain & EtOH (\%) & RS (\%) & FE (\%) \\
\hline F-707 & 9.2 & 3.8 & 75.4 \\
FA-91 & 9.7 & 3.3 & 79.5 \\
FF-725 & 9.9 & 2.9 & 81.2 \\
F-235 & 9.5 & 3.4 & 77.9 \\
F-25 & 9.7 & 3.2 & 79.5 \\
FK-727 & 10.5 & 2.3 & 86.1 \\
FC-620 & 9.9 & 2.8 & 81.2 \\
FH-620 & 9.7 & 3.3 & 79.5 \\
FAT-12 & 9.3 & 3.6 & 76.2 \\
F-514 & 10.4 & 2.3 & 85.3 \\
\hline
\end{tabular}

$\mathrm{EtOH}$, Ethanol yield; RS, residual sugars; FE, fermentation efficiency.

concentration by evaporation, and yeast and energy (Ryznar-Luty et al., 2008; Karlsson et al., 2013). No one has found convenient and economical disposal solution for vinasse. In this paper, we worked on the alcoholic fermentation of sugar cane molasses and studied the possibility of applying vinasse, instead of water in the preparation of the fermentation medium. We applied different vinasse percentages in the medium.

\section{MATERIALS AND METHODS}

\section{Sugarcane molasses}

Sugarcane molasses produced by Egyptian Sugar and Integrated Industries Company is used as carbon source for ethanol production in the distillation factories in El-Hawamdia-Giza- Egypt.

\section{Vinasse}

Vinasse containing $8.6 \%$ total solids and $1.34 \%$ fermentable sugars was taken from the output of distillery factories, El-HawamdiaEgyptian Sugar and Integrated Industries Company, Giza- Egypt.

\section{Yeast strains}

S. cerevisiae strains were obtained from Microbial Chemistry Department National Research Centre, Dokki, Cairo Egypt. The strains were subcultured on yeast extract, malt extract, peptone agar (YMPA) medium and preserved in refrigerator at $4^{\circ} \mathrm{C}$

\section{Inoculums preparation}

$100 \mathrm{ml}$ of medium ( $\mathrm{g} / \mathrm{L}$ ) consisting of malt extract (3), yeast extract (3), peptone (5) and sucrose (30) was poured into $250 \mathrm{ml}$ sterile conical flasks and steam sterilized at $121^{\circ} \mathrm{C}$ for $15 \mathrm{~min}$; it was cooled to room temperature, then inoculated with a loop of the selected yeast strains and incubated statistically at $34^{\circ} \mathrm{C}$ for $24 \mathrm{~h}$. The growth yeast containing $4 \times 10^{6} \mathrm{CFU} / \mathrm{ml}$ was used to inoculate the experimental flasks at $10 \%(\mathrm{v} / \mathrm{v})$.

\section{Experimental}

All laboratory studies were carried in $500 \mathrm{ml}$ capacity conical flasks that contained $200 \mathrm{ml}$ molasses fermentation medium with $20 \%$ fermentable sugars, non- adjustable $\mathrm{pH}$ at $36^{\circ} \mathrm{C}$.

\section{Screening of yeast strains}

Ten (10) yeast strains of $S$. cervisiae (Table 1) were cultured in diluted molasses medium ( $20 \% \mathrm{w} / \mathrm{v}$ fermentable sugars); no nutrients were added, to select the proper strains.

\section{Effect of replacing water with varying levels of vinasse}

The selected yeast strain was cultured in molasses medium diluted with vinasse instead of water at different levels (10, 20, 30, 40, 50, $60,70,80,90$ and $100 \%$ ) to study the effect of vinasse level on ethanol yield and fermentation efficiency.

\section{Effect of nitrogen source and level}

Three nitrogen sources (urea, ammonium sulfate and diammonium phosphate) were introduced individually in molasses medium diluted with different levels of vinasse to the equivalent of nitrogen at three concentrations each $(100,150$ and $200 \mathrm{ppm})$ to study their effect on ethanol yield and fermentation efficiency in sugar cane molasses medium.

\section{Effect of orthophosphoric acid supplementation}

Varying concentrations of orthophosphoric acid (25-75 ppm) were added to the fermentation.

\section{Effect of magnesium sulfate supplementation}

Varying concentrations of magnesium sulfate (15-45 ppm) were added to the fermentation media under the applied concentrations of urea and orthophosphoric acid.

\section{Effect of inoculums size}

Inoculums sizes ranging from $10-25 \% \mathrm{v} / \mathrm{v}$ were applied to study their effect on the final ethanol yield and FE in molasses medium diluted with different vinasse levels instead of water.

\section{Effect of fermentation time}

Varying percents of vinasse under optimized parameters of urea, orthophosphoric acid and magnesium sulfate and inoculum size were applied to investigate their effect on fermentation period for maximum ethanol production.

\section{Fermentation process}

Batch culture system was employed for the fermentation of diluted molasses with varying levels of vinasse instead of water in fermenters having working volume of $65 \mathrm{~m}^{3}$. They were supplemented with the parameters to be optimized, but afterwards a diluted molasses ( $20 \%$ fermentable sugars) was fed to the fermenters to enable yeast cells to utilize sugars in the molasses for conversion into ethanol. Batch molasses was adjusted so that fermenter vessels were filled to working capacity $\left(65 \mathrm{~m}^{3}\right)$, and then allowed to achieve complete fermentation. 
Table 2. Effect of recycling varying vinasse amounts in sugar cane molasses medium on the alcoholic fermentation yield and efficiency.

\begin{tabular}{cccc}
\hline Vinasse (\%, v/v) & EtOH (\%) & RS (\%) & FE (\%) \\
\hline 0 & 11 & 1.8 & 100 \\
10 & 11 & 1.9 & 100 \\
20 & 11.1 & 1.9 & 100.9 \\
30 & 11 & 2.1 & 100 \\
40 & 10.7 & 2.2 & 97.3 \\
50 & 10.5 & 2.3 & 95.5 \\
60 & 10.2 & 2.5 & 92.7 \\
70 & 9.6 & 3 & 87.3 \\
80 & 9.3 & 3.8 & 84.6 \\
90 & 8.9 & 4.4 & 80.9 \\
100 & $8 . .5$ & 4.9 & 77.3 \\
\hline
\end{tabular}

$\mathrm{EtOH}$, Ethanol yield; RS, residual sugars; FE, fermentation efficiency.

\section{Analytical procedures}

The sugar concentration was determined by rapid method. $5 \mathrm{ml}$ of fermented sample was taken and dissolved in $100 \mathrm{ml}$ of distilled water and mixed with $5 \mathrm{ml}$ of conc. $\mathrm{HCL}$ acid; it was heated at $70^{\circ} \mathrm{C}$ for a period of $10 \mathrm{~min}$. The obtained sample was neutralized by adding $\mathrm{NaOH}$, prepared up to $1000 \mathrm{ml}$ and transferred into burette solution. $5 \mathrm{ml}$ of Fehling $A$ and $5 \mathrm{ml}$ of Fehling $B$ were taken and mixed with 10 to $15 \mathrm{ml}$ of distilled water in a conical flask and methylene blue indicator was added. The conical flask solution was titrated with burette solution in boiling conditions until disappearance of blue color. The sugar concentration was calculated by using the formula: (sugar concentration $(\mathrm{gm} / \mathrm{l})=$ [(dilution factor $\times$ Fehling factor) / titrate value] $\times 100)$. Cells count (CFU) were determined using microscope with the help of haemocytometer. Cell viability was checked by using methylene blue indicator. The dead cells were stained with blue indicator while viable cells remained uncolored. Ethanol yield $(\mathrm{EtOH} \% \mathrm{v} / \mathrm{v})$ of the fermented samples was measured by ebulliometer approved in distillation factories (Fadel et al., 2013): theoretical yield (total reducing sugars $\mathrm{g} / \mathrm{l} \times 0.51 \times 0.79)$ multiplied by 100 . The relative fermentation efficiency (RF) of fermentation process determined the percent of ethanol yield when vinasse was used in relation to the percent of ethanol yield in the molasses medium without diluted vinasse $(100 \%)$.

\section{Reproducibility of the results}

All experiments were run at least three times and all reported data are given in mean values.

\section{RESULTS AND DISCUSSION}

\section{Selection of yeast strain}

Yeast strains are limiting factor in fermentation process. As a result, high osmo-tolerant $S$. cerevisiae strains are needed to ferment high concentrate molasses. This would save water for molasses dilution and reduce vinasse produced (Ingledew and Bellissimi, 2012). Ten (10) yeast strains of $S$. cerevisiae were screened in molasses medium diluted with $50 \%$ vinasse instead of water to select the most tolerant (Table 1). Data show that S. cerevisiae F-727 and F-514 are more efficient than the other tested strains for producing ethanol in molasses medium diluted with vinasse; they produced $10.8 \% \mathrm{v} / \mathrm{v}$ and $10.4 \mathrm{v} / \mathrm{v}$, respectively. Fadel (2013) showed that the selection of yeast strain is one of the solutions to the problem of vinasse. This is because the selection of higher ethanol yielding yeast strain and optimization of the fermentation parameters improved both yield and economics of the fermentation process (Fadel et al., 2013). Research on ethanol fermentation, including the search for efficient $S$. cerevisiae strains has been on for years in order to lower down the cost of production. Desired yeast strains have the special property of possessing particularly efficient aerobic and anaerobic metabolic capabilities, making them high ethanol producers. They could also possess other industrially-important properties such as ethanol tolerance, thermotolerance and resistance to killer yeasts (Irene et al., 2009). S. cerevisiae F-727 was selected for further studies.

\section{Effect of vinasse amount in fermentation medium}

Vinasse replaced water in different levels for dilution molasses for obtaining desired sugars concentration in fermentation molasses medium (Table 2). Results show that up to $30 \% \mathrm{v} / \mathrm{v}$ replaced vinasse instead of water; there is no effect on ethanol yield or fermentation efficiency. There was slight efficiency when $40 \% \mathrm{v} / \mathrm{v}$ vinasse was used instead of water. There was relative decrease in ethanol yield and fermentation efficiency when vinasse was introduced above $50 \% \mathrm{v} / \mathrm{v}$ instead of water in the fermentation medium; but it increased as the level of vinasse increased. From the results obtained, it is suggested that vinasse should be increased instead of water in the fermentation medium and also to increase the inhibitors involved in the end fermentation whose vinasse is tacked from methanol, fusel alcohols, acetic acid, aldehides and aromatic compounds (Arshad et al. 2008). Heavy metals in the fermentation medium should be increased and as well as one or more minerals toxic to biomass production or their activity (Madaree et al., 1991). Other by-products of the metabolism of the yeast like glicerol, propanol, furfural and lactic acid also inhibit the fermentation and growth speed (Navarro et al., 2000). Besides the inhibitory effect of the fermentation byproducts, the effect of the increased solids on the vinasse is very important, including the non-fermentable sugars remaining in the vinasse after fermentation and distillation. Mineral salts not assimilated by the yeast also increase with the increased vinasse fermentations, due to the molasses and the nutrients used for preparing the medium of fermentation (Maiorella et al., 1984). 
Table 3. Effect of urea supplementation on the ethanol yield production from sugar cane molasses diluted with different amounts of vinasse instead of water by S. cerevisiae F-727.

\begin{tabular}{ccccccccc}
\hline \multirow{2}{*}{ Vinasse (\%, v/v) } & \multicolumn{7}{c}{ Urea concentration (ppm N/I) } \\
\cline { 2 - 9 } & \multicolumn{7}{c}{-} & \multicolumn{7}{c}{$\mathbf{1 0 0}$} & \multicolumn{2}{c}{$\mathbf{2 0 0}$} & \multicolumn{2}{c}{$\mathbf{3 0 0}$} \\
\cline { 2 - 8 } & EtOH (\%) & FE (\%) & EtOH (\%) & FE (\%) & EtOH (\%) & FE (\%) & EtOH (\%) & FE (\%) \\
\hline 0 & 11.0 & 100.0 & 11.42 & 100 & 11.46 & 100.0 & 11.34 & 100.0 \\
10 & 11.0 & 100.0 & 11.42 & 100 & 11.46 & 100.0 & 1.341 & 100.0 \\
20 & 11.1 & 100.1 & 11.46 & 100.4 & 11.46 & 100.0 & 11.28 & 99.5 \\
30 & 11.0 & 100.0 & 11.38 & 99.7 & 11.36 & 99.1 & 1.261 & 99.4 \\
40 & 10.9 & 99.9 & 11.22 & 98.3 & 11.12 & 97.4 & 11.10 & 97.9 \\
50 & 10.5 & 98.2 & 11.06 & 96.9 & 11.10 & 97.2 & 10.94 & 96.5 \\
60 & 10.6 & 96.4 & 10.72 & 93.9 & 10.76 & 94.2 & 10.68 & 94.2 \\
70 & 10.3 & 93.7 & 10.40 & 91.1 & 10.22 & 89.5 & 10.42 & 91.9 \\
80 & 9.9 & 90.0 & 10.08 & 88.3 & 10.09 & 88.4 & 9.98 & 88.0 \\
90 & 9.6 & 87.3 & 9.77 & 85.6 & 9.84 & 86.2 & 9.68 & 85.4 \\
100 & 9.5 & 86.4 & 9.40 & 82.3 & 9.46 & 82.8 & 9.50 & 83.8 \\
\hline
\end{tabular}

EtOH, Ethanol yield; FE, fermentation efficiency.

\section{Effect of nitrogen sources}

\section{Urea}

Urea was used as cheap nitrogen source in producing alcoholic fermentation. Table 3 shows that it enhanced the ethanol yield as well as the fermentation efficiency (FE) compared to the medium free from nitrogen source. More enhancement was achieved when $150 \mathrm{ppm}$ of urea was introduced in the molasses fermentation medium diluted with water and $100 \mathrm{ppm}$ of urea in the molasses fermentation medium diluted with vinasse. From this, the yeast strain can utilize the residual nitrogen involved in vinasse. There is the possibility of saving an amount of nitrogen when vinasse is recycled as diluents in fermentation medium. Nitrogen deficiency slows down yeast growth and the fermentation efficiency (Dukes, 1991), due to the inhibition of the synthesis of protein transporting sugar through the cell membrane to the interior of the cells. It has been shown that an adequate nitrogen increases yeast growth provided the other essential yeast nutrients are not lacking (Nofemele et al., 2012). Guojun et al. (2012) concluded that production of high levels of ethanol could be achieved by supplementing urea as nitrogen source during ethanol fermentation.

\section{Effect of diammonium phosphate (DAP)}

Diammonium phosphate was used as good nitrogen and phosphorus sources in fermentation medium for ethanol yield (Arshad et al., 2008). Table 4 shows that adding $300 \mathrm{mg} \mathrm{Nl}^{-1}$ of DAP to the fermentation medium was more suitable than low or high concentrations. Arrizon and Gschaedle (2002) evaluated the effect of assimilable nitrogen on growth in batch cultures of $S$. cerevisiae under different nitrogen concentrations (from 16.5 to $805 \mathrm{mg} \mathrm{N}^{-1}$ ). They showed that lower than $66 \mathrm{mg} \mathrm{N} \mathrm{I}^{-1}$ slowed down cell growth and a relevant decrease in cell biomass was observed. In the strain, it appears the highest biomass production was observed in the media with $402 \mathrm{mg} \mathrm{N} \mathrm{I}^{-1}$. The kinetics of glucose and nitrogen consumption indicates that the ability of the yeast strain to break down sugars is strongly connected with increased nitrogen availability in the media. The results show that supplementation with diammonium phosphate added during fermentation increased cell population, fermentation rate and ethanol yield. The ammonium ion also serves as an allosteric regulator for one of the enzymes used in glycolysis and may also have an effect on how the yeast cell transports glucose and fructose into the cell (Arrizon and Gschaedler, 2002).

\section{Effect of ammonium sulfate}

Fermentation rate and the time required for completion of the alcoholic fermentation are strongly dependent on nitrogen availability. At the stationary phase, the addition of nitrogen source is effective in increasing cell population, fermentation rate and ethanol yield (FerreiraMendes et al., 2004). The obtained data revealed that the addition of ammonium sulfate to fermentation medium at any level resulted in decrease in ethanol yield by $S$. cerevisiae F-727. Our finding agrees with previous studies which show that there were significant differences in the amount of ethanol produced when ammonium sulfate was added to the fermentation medium, although this depended on the yeast strain used. When assimilable nitrogen was added, ethanol production 
Table 4. Effect of diammonium phosphate supplementation on the ethanol yield production from sugar cane molasses diluted with different amounts of vinasse instead of water by S.cerevisiae F-727.

\begin{tabular}{ccccccccc}
\hline \multirow{2}{*}{ Vinasse (\%, v/v) } & \multicolumn{7}{c}{ Diammonium phosphate concentration (ppm N/I) } \\
\cline { 2 - 8 } & \multicolumn{7}{c}{-} & \multicolumn{2}{c}{$\mathbf{1 0 0}$} & \multicolumn{3}{c}{$\mathbf{1 5 0}$} & \multicolumn{2}{c}{$\mathbf{2 0 0}$} \\
\cline { 2 - 8 } & EtOH (\%) & FE (\%) & EtOH (\%) & FE (\%) & EtOH (\%) & FE (\%) & EtOH (\%) & FE (\%) \\
\hline 0 & 11.0 & 100.0 & 11.62 & 100.0 & 11.82 & 100 & 11.80 & 99.2 \\
10 & 11.1 & 100.0 & 11.62 & 100.0 & 11.82 & 100 & 1.701 & 99.2 \\
20 & 11.1 & 100.1 & 11.72 & 100.7 & 11.82 & 100 & 11.70 & 98.6 \\
30 & 11.0 & 100.0 & 11.82 & 101.7 & 11.62 & 98.3 & 1.641 & 95.8 \\
40 & 10.9 & 99.91 & 11.82 & 101.7 & 11.52 & 97.5 & 11.30 & 95.3 \\
50 & 10.8 & 98.23 & 11.62 & 100.0 & 11.44 & 96.8 & 11.24 & 93.2 \\
60 & 10.6 & 96.42 & 11.44 & 98.5 & 11.29 & 95.2 & 11.00 & 91.7 \\
70 & 10.3 & 93.71 & 11.12 & 95.7 & 11.02 & 93.2 & 10.82 & 86.8 \\
80 & 9.9 & 90.00 & 10.82 & 93.1 & 10.88 & 92.1 & 10.24 & 85.3 \\
90 & 9.6 & 87.32 & 10.27 & 88.4 & 10.26 & 86.8 & 10.06 & 83.6 \\
100 & 9.5 & 9.96 & 9.96 & 85.7 & 9.96 & 84.3 & 9.86 & 100 \\
\hline
\end{tabular}

EtOH, Ethanol yield; FE, fermentation efficiency.

Table 5. Effect of ammonium sulfate supplementation on the ethanol yield production from sugar cane molasses diluted with different amounts of vinasse instead of water by S. cerevisiae F-727.

\begin{tabular}{ccccccccc}
\hline & \multicolumn{7}{c}{ Diammonium phosphate Concentration (ppm N/I) } \\
\cline { 2 - 9 } Vinasse (\%, v/v) & \multicolumn{7}{c}{$\mathbf{1 0 0}$} & \multicolumn{3}{c}{$\mathbf{1 5 0}$} & \multicolumn{2}{c}{$\mathbf{2 0 0}$} \\
\cline { 2 - 9 } & EtOH (\%) & FE (\%) & EtOH (\%) & FE (\%) & EtOH (\%) & FE (\%) & EtOH (\%) & FE (\%) \\
\hline 0 & 11.0 & 100.0 & 11.02 & 100 & 10.92 & 100 & 10.80 & 100 \\
10 & 11.1 & 100.0 & 11.00 & 99.9 & 10.90 & 100 & 10.80 & 100 \\
20 & 11.1 & 100.1 & 10.96 & 99.5 & 10.90 & 100 & 10.76 & 99.6 \\
30 & 11.0 & 100.0 & 10.90 & 98.9 & 10.58 & 96.9 & 10.46 & 96.9 \\
40 & 10.9 & 99.91 & 10.68 & 96.9 & 10.42 & 95.4 & 10.22 & 94.6 \\
50 & 10.8 & 98.23 & 10.62 & 96.4 & 10.38 & 95.1 & 10.18 & 94.3 \\
60 & 10.6 & 96.42 & 10.58 & 96.0 & 10.30 & 94.3 & 10.08 & 93.3 \\
70 & 10.3 & 93.71 & 10.50 & 95.3 & 10.00 & 91.6 & 9.96 & 92.2 \\
80 & 9.9 & 90.00 & 10.48 & 95.1 & 9.26 & 84.8 & 9.16 & 84.8 \\
90 & 9.6 & 87.32 & 9.66 & 87.7 & 9.26 & 84.8 & 9.06 & 83.9 \\
100 & 9.5 & 9.96 & 9.36 & 84.9 & 9.04 & 82.8 & 9.00 & 83.3 \\
\hline
\end{tabular}

EtOH, Ethanol yield; FE, fermentation efficiency.

either increased (with S. cerevisiae AR2 and S. cerevisiae NT116 yeast strains) or decreased (with $S$. cerevisiae LW LVCB CT1+ yeast (Hernandez-Orte et al., 2006). Also, nitrogen added in the form of ammonium sulfate did not affect the ethanol production rate (Arshad et al., 2011).

Table 5 shows the effect of ammonium sulfate supplementation on the ethanol yield obtained from sugar cane molasses diluted with different amounts of vinasse instead of water by S. cerevisiae F-727.

\section{Effect of orthophpsphoric acid (OPA) supplementation}

Table 6 shows that the supplementation of OPA plus urea in fermentation medium enhanced ethanol yield and raised the possibility of applying vinasse in fermentation medium up to $40 \%$ instead of water. Data shows also that maximum ethanol yield can be obtained by using low amount of OPA in the case of fermentation molasses medium diluted with vinasse. Nitrogen and phosphorus are the main nutritional requirements for yeast growth and maximum ethanol production efficiency. Although molasses contains most of the nutrients required for yeast growth, generally nitrogen and phosphate are added to enhance yeast growth and ethanol production (Malherbe et al., 2007). For optimum yeast efficiency in molasses medium, OPA was used as phosphate source. Phosphorus plays the major role in the glycolysis cycle in yeast cell. Extensive studies were previously performed 
Table 6. effect of orthophosphoric acid supplementation on the ethanol yield production from sugar cane molasses diluted with different amounts of vinasse instead of water by S.cerevisiae F-727.

\begin{tabular}{ccccccccc}
\hline & \multicolumn{7}{c}{ Orthophosphoric acid (ppm) } \\
\cline { 2 - 9 } Vinasse (\%, v/v) & \multicolumn{7}{c}{$\mathbf{2 5}$} & \multicolumn{2}{c}{$\mathbf{5 0}$} & \multicolumn{2}{c}{$\mathbf{7 5}$} \\
\cline { 2 - 9 } & EtOH (\%) & FE (\%) & EtOH (\%) & FE (\%) & EtOH (\%) & FE (\%) & EtOH (\%) & FE (\%) \\
\hline 0 & 11.42 & 100.0 & 11.72 & 100.0 & 11.78 & 100 & 11.70 & 100 \\
10 & 11.42 & 100.0 & 11.72 & 100.0 & 11.78 & 100 & 1.701 & 100 \\
20 & 11.46 & 100.1 & 11.82 & 100.2 & 11.82 & 100.2 & 11.72 & 100.2 \\
30 & 11.38 & 100.0 & 11.70 & 99.9 & 11.72 & 99.5 & 1.601 & 99.2 \\
40 & 11.22 & 99.91 & 11.46 & 97.8 & 11.52 & 97.8 & 11.36 & 97.1 \\
50 & 11.06 & 98.23 & 11.22 & 95.7 & 11.12 & 94.4 & 11.24 & 96.1 \\
60 & 10.72 & 96.42 & 11.14 & 95.1 & 11.04 & 93.7 & 11.00 & 94.0 \\
70 & 10.40 & 93.71 & 10.70 & 91.3 & 10.72 & 91.0 & 10.92 & 93.3 \\
80 & 10.08 & 90.00 & 10.32 & 88.1 & 10.18 & 86.9 & 10.54 & 90.1 \\
90 & 9.77 & 87.32 & 9.96 & 85.0 & 9.96 & 84.6 & 10.26 & 87.7 \\
100 & 9.40 & 9.96 & 9.72 & 82.9 & 9.76 & 82.9 & 9.86 & 84.3 \\
\hline
\end{tabular}

EtOH, Ethanol yield; FE, fermentation efficiency.

Table 7. Effect of magnesium sulfate supplementation on the ethanol yield production from sugar cane molasses diluted with different amounts of vinasse instead of water by S. cerevisiae F-727.

\begin{tabular}{ccccccccc}
\hline & \multicolumn{7}{c}{ Magnesium sulfate concentration (ppm) } \\
\cline { 2 - 8 } Vinasse (\%, v/v) & \multicolumn{7}{c}{ - } & \multicolumn{3}{c}{$\mathbf{3 0}$} & \multicolumn{4}{c}{$\mathbf{4 5}$} \\
\cline { 2 - 8 } & EtOH (\%) & FE (\%) & EtOH (\%) & FE (\%) & EtOH (\%) & FE (\%) & EtOH (\%) & FE (\%) \\
\hline 0 & 11.72 & 100.0 & 11.88 & 100 & 12.00 & 100 & 12.00 & 100 \\
10 & 11.78 & 100.1 & 11.88 & 100.1 & 12.00 & 100 & 12.00 & 100 \\
20 & 11.82 & 100.1 & 11.94 & 100.1 & 12.08 & 100 & 12.00 & 100 \\
30 & 11.70 & 100.0 & 12.00 & 100.1 & 12.12 & 100 & 12.08 & 98.5 \\
40 & 11.46 & 99.91 & 11.82 & 100.2 & 12.00 & 100 & 11.90 & 97.8 \\
50 & 11.22 & 98.23 & 11.22 & 100 & 11.34 & 99.2 & 11.32 & 94.6 \\
60 & 11.14 & 96.42 & 11.04 & 99.6 & 11.40 & 98.8 & 11.04 & 93.1 \\
70 & 10.60 & 93.71 & 10.80 & 97.2 & 11.62 & 98.2 & 10.70 & 90.2 \\
80 & 10.32 & 90.00 & 10.42 & 92.3 & 10.46 & 95.3 & 10.44 & 88.0 \\
90 & 9.96 & 87.32 & 9.86 & 89.8 & 9.36 & 92.4 & 10.16 & 85.7 \\
100 & 9.42 & 86.41 & 9.22 & 87.1 & 9.36 & 90.1 & 9.22 & 77.7 \\
\hline
\end{tabular}

EtOH, Ethanol yield; FE, fermentation efficiency.

to optimize nitrogen and phosphorous sources and other supplements (Pretorius et al., 2013). Higher ethanol production has also been previously reported with urea and phosphoric acid, making the process very economical (Arshad et al., 2008).

\section{Effect of varying concentrations of magnesium sulfate}

Varying concentrations of magnesium sulfate were supplemented in fermentation medium diluted with different percents of vinasse compared to those diluted with fresh water under the above optimized nutrients levels of urea and orthophosphoric acid (Table 7). Results show that ethanol yield as well as relative fermentation efficiency was enhanced by introducing magnesium sulfate in molasses medium diluted with vinasse, especially in the medium diluted with vinasse above $40 \%$. The data shows that the demand level of magnesium sulfate was increased in the medium diluted with vinasse than that diluted with water; this led to maximum ethanol production. Also, the addition of magnesium enabled yeast strain to utilize some fermentable sugars involved in vinasse to yield more ethanol than that diluted with fresh water. The data agree 
Table 8. Effect of varying inoculum size on ethanol yield from sugar cane molasses diluted with different amounts of vinasse instead of water by S.cerevisiae F-727.

\begin{tabular}{ccccccccc}
\hline & \multicolumn{7}{c}{ Inoculum size (\%, v/v) } \\
\cline { 2 - 8 } Vinasse (\%, v/v) & \multicolumn{7}{c}{$\mathbf{1 0}$} & \multicolumn{2}{c}{ 15 } & \multicolumn{2}{c}{ 20 } \\
\cline { 2 - 9 } & EtOH (\%) & FE (\%) & EtOH (\%) & FE (\%) & EtOH (\%) & FE (\%) & EtOH (\%) & FE (\%) \\
\hline 0 & 12.00 & 100 & 12.00 & 100 & 12.00 & 100.0 & 11.98 & 100.0 \\
10 & 12.00 & 100 & 12.00 & 100 & 12.00 & 100.0 & 11.98 & 100.0 \\
20 & 12.08 & 100 & 12.00 & 100 & 12.00 & 100.0 & 11.98 & 100.0 \\
30 & 12.12 & 100 & 12.08 & 100.7 & 12.08 & 100.7 & 12.18 & 101.7 \\
40 & 12.00 & 97.8 & 12.22 & 101.8 & 12.28 & 102.3 & 12.32 & 102.8 \\
50 & 1174 & 96.7 & 12.02 & 100.2 & 12.12 & 101.0 & 12.22 & 102.0 \\
60 & 11.60 & 95.0 & 11.76 & 98.00 & 11.81 & 98.4 & 11.78. & 98.3 \\
70 & 10.40 & 85.9 & 11.64 & 97.00 & 11.62 & 96.8 & 11.60 & 96.8 \\
80 & 10.26 & 82.2 & 10.70 & 89.20 & 10.96 & 91.3 & 11.00 & 91.8 \\
90 & 9.86 & 80.5 & 10.24 & 85.30 & 10.52 & 87.7 & 10.65 & 88.9 \\
100 & 9.66 & 100 & 9.72 & 81.00 & 9.84 & 82.0 & 9.90 & 82.6 \\
\hline
\end{tabular}

EtOH, Ethanol yield; FE, fermentation efficiency.

with scientific knowledge that vinasse contains high level of potassium which inhibits the metabolism of magnesium. As a result, more magnesium is needed to overcome this problem. Deficiencies and imbalances in minerals and cations serving as co-factors for glycolytic and other enzymatic reactions can result in fermentation arrest (Blackwell et al., 1997). Magnesium plays a key role in metabolic control, growth and cell proliferation, glycolytic pathway and subsequently ethanol production (Walker, 1994).

\section{Effect of varying inoculum sizes}

Ethanol yield and production of co-products has a major relationship in ethanol fermentation. Extensive studies have been carried out to investigate the effect of yeast inoculation rate to help the yeast cells overcome the bacterial cells on the basis of size and number (Fadel et al., 2013). Effect of varying inoculum sizes on ethanol yield was studied under the above optimized parameters. Maximum ethanol content was found when the inoculation rate was $25 \% \mathrm{v} / \mathrm{v}$ and was $12.3 \% \mathrm{v} / \mathrm{v}$ in fermentation medium diluted with $50 \%$ vinasse instead of water (Table 8). The obtained results showed that yeast cells absorb heavy metals which inhibit the enzymatic system responsible for ethanol production by yeast cells, and as the number of cells increases the amount of heavy metals per cell decreases from the surrounding medium. In brewing, higher yeast inoculum causes attenuation to initiate the process more rapidly, and reduces viability losses that occur immediately after pitching. In a previous study, ethanol yield increased with increasing inoculum size and yield of methanol, and aldehydes were the lowest when inoculum size was above $30 \%$ (Arshad et al., 2008).

\section{Effect of dilution with vinasse on fermentation time course}

Table 9 shows that the fermentation medium diluted with above $30 \%(\mathrm{v} / \mathrm{v})$ increased the fermentation time, leading to the production of the highest ethanol in the fermentation mash. It can be said that the inhibitory effect of heavy meals (Arshad et al., 2008) as well as osmoses effect increased with the amount of vinasse added to the fermentation medium (Patrascu et al., 2009).

\section{Industrial application}

Vinasse $[25 \%(\mathrm{v} / \mathrm{v})]$ was applied instead of water in diluted fermentation medium as well as $100 \%(\mathrm{v} / \mathrm{v})$ in industrial fermenters of $65 \mathrm{~m}^{3}$ capacity. Table 10 shows the safe means of using vinasse obtained from the fermentation of sugar cane molasses for ethanol production in distillery factories without affecting both ethanol yield and fermentation efficiency (FE). Also, the yeast can partially utilize some residual fermented sugars involved in vinasse, and this has the advantage of raising the distillery factory efficiency.

\section{Conclusion}

This study showed the safe possibility of using up to $50 \%$ vinasse resulting from the fermentation of sugar cane molasses for ethanol production instead of fresh water in distillery factories without affecting both ethanol yield and fermentation efficiency as well as the yeast can partially utilized some of residual fermented sugars and nutrients involved in vinasse and this has advantage in rising the distillery factory efficiency causing low ethanol cost production. On the other hand aids in the solution of the environmental problem for vinasse disposal. 
Table 9. effect of dilution with vinasse on fermentation time course on ethanol yield from sugar cane molasses diluted with different amounts of vinasse instead of water by S.cerevisiae F-727.

\begin{tabular}{cllllllll}
\hline \multirow{2}{*}{ Vinasse (\%, v/v) } & \multicolumn{7}{c}{ Fermentation time (h) } \\
\cline { 2 - 9 } & \multicolumn{2}{c}{$\mathbf{2 4}$} & \multicolumn{7}{c}{$\mathbf{3 0}$} & \multicolumn{3}{c}{$\mathbf{3 6}$} & \multicolumn{2}{c}{$\mathbf{4 2}$} \\
\hline & EtOH (\%) & RS (\%) & EtOH (\%) & RS (\%) & EtOH (\%) & RS (\%) & EtOH (\%) & RS (\%) \\
\hline 0 & 11.20 & 3.30 & 11.88 & 1.74 & 11.98 & 1.64 & 11.94 & \\
10 & 11.20 & 3.31 & 11.88 & 1.75 & 11.98 & 1.64 & 11.94 & 1.64 \\
20 & 11.16 & 3.77 & 11.90 & 1.76 & 11.98 & 1.76 & 11.98 & 1.75 \\
30 & 11.04 & 4.37 & 12.08 & 1.86 & 12.18 & 1.96 & 12.28 & 1.76 \\
40 & 11.02 & 4.40 & 12.32 & 2.10 & 12.32 & 2.10 & 12.36 & 186 \\
50 & 11.02 & 4.41 & 12.02 & 2.82 & 12.22 & 2.68 & 12.24 & 2.06 \\
60 & 10.90 & 4.64 & 11.32 & 3.36 & 11.78 & 3.26 & 11.86 & 2.64 \\
70 & 10.74 & 5.20 & 11.08 & 4.64 & 11.60 & 4.64 & 11.60 & 3.16 \\
80 & 10.60 & 5.48 & 10.20 & 5.44 & 11.00 & 5.44 & 11.0 & 4.64 \\
90 & 10.22 & 6.28 & 10.44 & 6.12 & 10.65 & 5.92 & 10.65 & 5.44 \\
100 & 9.72 & $\mathbf{7 . 0 8}$ & 9.90 & 7.00 & 9.94 & 6.98 & 9.98 & 5.92 \\
\hline
\end{tabular}

EtOH, Ethanol yield; FE, fermentation efficiency.

Table 10. Effect of dilution with vinasse instead of water on ethanol yield and fermentation efficiency from sugar cane molasses by S. cerevisiae F-727 .in Hawamdia Distillation Factories.

\begin{tabular}{cccccc}
\hline Vinasse (\%) & TFS (\%) & pH & EtOH (\%) & RS (\%) & FE (\%) \\
\hline 0 & 17.22 & 4.85 & 9.10 & 2.44 & 87.6 \\
5 & 17.24 & 4.85 & 9.10 & 2.22 & 87.6 \\
10 & 17.28 & 4.76 & 9.12 & 2.46 & 87.6 \\
15 & 17.36 & 4.78 & 9.15 & 2.52 & 87.5 \\
25 & 17.48 & 4.89 & 9.20 & 2.76 & 87.2 \\
100 & 17.86 & 4.90 & 7.90 & 5.64 & 73.7 \\
\hline
\end{tabular}

Total fermented sugars (TFS); EtOH, Ethanol yield; RS, residual sugars; FE, fermentation efficiency.

\section{Conflict of Interests}

The author(s) have not declared any conflict of interest.

\section{ACKNOWLEDGEMENTS}

The authors deeply thank Engineer H. K. Hassan, Chairman and Managing Director of Egyptian Sugar and Integrated Industry Company. Also all thanks to the Chairman and members of Hawamdia Distillation Factories.

\section{REFERENCES}

Aparecida C, Janaina C, Escher, Correia JE, Marinho JFU, Fontanett CS (2013). Sugarcane vinasse: Environmental implications of its use Waste Manage. 33(12):2752-276.

Arrizon J, Gschaedler A (2002). Increasing fermentation efficiency at high sugar concentrations by supplementing an additional source of nitrogen during the exponential phase of the tequila fermentation process. Can. J. Microbiol. 48:965-970.

Arshad M, Khan ZM, Khalil-ur-Rehman, Shah FA, Rajoka MI (2008).Optimization of process variables for minimization of byproduct formation during fermentation of blackstrap molasses to ethanol at industrial scale. Lett . Appl . Microbiol. 47(5):410-414.

Arshad M, Anjum ZM, Asghar M, HaqNawaz B (2011). Improving bioethanol yield: Using virginiamycin and sodium flouride at a Pakistani distillery. Afr. J. Biotechnol.10 (53):11071-11074.

Baez-Smith C (2006). Anaerobic Digestion of Vinasse for the Production OfMethane in the Sugar Cane Distillery2006 SPRI Conference on Sugar Processing Loxahatchee, Florida, USA.

Blackwell KJ, Tobin JM and Avery SV (1997). Mangenese uptake and toxicity in magnesium supplemented and unsupplemented Saccharomyces cerevisiae . Appl. Microbiol. Biotechnol. 47:180-184.

Dukes B, Goldspink B, Elliott J, Frane R (1991). Time of nitrogenous fertilization cane reduce fermentation time and improve wine quality. Am. Soc. Enol. Vitic., Davis, CA. pp. 249-254.

Fadel M (2013). Production of fuel ethanol from sugar cane molasses. Microbes process Chapter 4, pp. 93-115.

Fadel M, Abeer AK, Foukia EM, Tarek K (2013) .High level Ethanol from Sugar Cane Molasses by Anew Thermotolerant Saccharomyces cervisiae Strain in industrial Scale. Biotechnol. Res. Int. vol.2013 ID253286 6 pages.

Ferreira-Mendes A, Mendes-Faia A, Leao C (2004). Growth and fermentation patterns of Saccharomyces cerevisiae under different ammonium concentrations and its implications in winemaking industry. J. Appl. Microbiol. 97(3):540-5.

Guojun Yue, Jianliang Yu, Xu Zhang Tianwei Tan (2012), The influence of nitrogen sources on ethanol production by yeast from concentrated sweet sorghum juice. Biomass Bioenergy 39:48-55. 
Hernandez-Orte P, Bely M, Cacho J, Ferreira V (2006), Impact of ammonium additions on volatile acidity, ethanol, and aromatic compound production by different Saccharomyces cerevisiae strains during fermentation in controlled synthetic media. Aust. J. Grape Wine Res. 12(2):150-160.

Ingledew WM, Bellissimi E (2012). Active dry,High- alcohol yeast: The new yeast on the block. Ethanol producer magazine. International fuel ethanol workshop and EXPO. June,4-7.

Irene G, Pajares Francisco B, Elegado Jose Paolo V, Magbanua Asuncion K. Raymundo (2009). Selection of High Ethanol-Producing Saccharomyces cerevisiae Strains, their Fermentation Properties, and Genetic Differentiation Based on rep-PCR. Philippine J. Sci. Vol.138 No. 1 June 2009.

Karlsson E, Gourdon M, Olausson L, Vamling L (2013). Heat transfer for falling film evaporation of black liquor up to very high. Int. J. Heat Mass Transf. 65:907-918

Madaree SJ, Rein PW, Wenman CM (1991). Investigation into problems ethanol fermentations. Proceeding of the South Africa Sugar Technologists Association, June 1991.

Maiorella B, Blanch H, Wilke C (1984). Feed and component inhibition in ethanol fermentation. Biotechnol. Bioeng. 26(10):1155-1156.

Malherbe S, Bauer FF, Du Toit M (2007).Understanding problem fermentation-A Review. S. Afr. J. Enol. Vitic. 28(2):169-185.

Navarro A, Seplveda M, Rubio M (2000). Bio-concentration of vinasse from alcoholic fermentation of sugar cane molasses. Waste Manage. 20:581-585.
Nofemele Z, Shukl P, Trussler A, Permaul K, Singh S (2012). Improvement of ethanol production from sugar cane molasses through enhanced nutrient supplementation using Saccharomyces cerevisiae. J. Brewing Distilling 3(2):29-35.

Patrascu E, Rapeanu G, Hopulele T (2009).Current approaches toeffecient biotechnological production of ethanol. Innov. Rom. Food Biotechnol. 4.1-11.

Pretorius IS, Toil M, Rensburg P (2013). Designer yeast for the fermentation industry of the 21 st century. Food Technol. Biotechnol. 41(1):3-10.

Ryznar-Luty A, Krzywonos M, Cibis E, Miśkiewicz T (2008) .Aerobic Biodegradation of Vinasse by a Mixed Culture of Bacteria of the Genus Bacillus: Optimization of Temperature, $\mathrm{pH}$ and Oxygenation State. Pol. J. Environ. Stud. 17(1):101-112.

Voegele E (2009). Brazilian researchers develop method to reduce volume of vinasse coproduct Ethanol producer magazine April

Walker GM (1994). The role of magnesium in biotechnology. Crit. Rev. Biotechnol. 14:311-354. 